\title{
Sequential effects of the proteasome inhibitor bortezomib and chemotherapeutic agents in uterine cervical cancer cell lines
}

\author{
YUICHIRO MIYAMOTO $^{1}$, SHUNSUKE NAKAGAWA ${ }^{3}$, OSAMU WADA-HIRAIKE ${ }^{1}$, \\ TAKAYUKI SEIKI $^{1}$, MICHIHIRO TANIKAWA ${ }^{1}$, HARUKO HIRAIKE $^{1}$, KENBUN SONE $^{1}$, \\ KAZUNORI NAGASAKA ${ }^{1}$, KATSUTOSHI ODA ${ }^{1}$, KEI KAWANA ${ }^{1}$, KEIICHI NAKAGAWA ${ }^{2}$, \\ TOMOYUKI FUJII $^{1}$, TETSU YANO ${ }^{1}$, SHIRO KOZUMA ${ }^{1}$ and YUJI TAKETANI ${ }^{1}$ \\ Departments of ${ }^{1}$ Obstetrics and Gynecology, and ${ }^{2}$ Radiology, Graduate School of Medicine, \\ The University of Tokyo, Tokyo 113-8655; ${ }^{3}$ Department of Obstetrics and Gynecology, \\ School of Medicine, Teikyo University, Tokyo 173-8605, Japan
}

Received August 8, 2012; Accepted September 10, 2012

DOI: 10.3892/or.2012.2072

\begin{abstract}
Although the prognosis of uterine cervical cancer has improved due to the advances of treatment modalities, survival of recurrent or metastatic cervical cancer remains poor. Cisplatin is an effective radiosensitizer, but its single agent activity in recurrent cervical cancer is disappointing. Inactivation of tumor suppressors through ubiquitin-mediated degradation by human papillomavirus is known to be a critical step in the carcinogenesis of uterine cervix. Bortezomib, a selective inhibitor of the proteasome, has been shown to inhibit the growth of several solid tumors. To determine the role of bortezomib in cervical cancer as a chemotherapeutic agent, we studied its biological properties. Bortezomib efficiently inhibited the proteasomal activities in cervical cancer cells, and an increased expression of tumor suppressors such as p53, $\mathrm{hDlg}$ and hScrib became evident. In addition, sequential or concomitant treatment of bortezomib and cisplatin stimulated the expression of p53, hScrib and p21 and the stimulation was markedly influenced by the order of drugs in HeLa cells. We further confirmed that the concomitant use of bortezomib and cisplatin has synergistic inhibitory effects on the growth of xenograft tumors derived from HeLa cells. Our data establish the possibility that the concomitant use of bortezomib and cisplatin could be an alternative choice in cases resistant to conventional chemotherapy, and sequential effects must be considered for advanced and therapy-resistant cervical cancer patients.
\end{abstract}

Correspondence to: Dr Shunsuke Nakagawa, Department of Obstetrics and Gynecology, School of Medicine, Teikyo University, 2-11-1 Kaga, Itabashi-ku, Tokyo 173-8605, Japan

E-mail: nakagawas-tky@umin.ac.jp

Key words: bortezomib, cell growth, cervical cancer, cisplatin, combination therapy, proteasome inhibitor

\section{Introduction}

Cancer of the uterine cervix is the second most common cause of gynecologic cancer mortality worldwide, and it is reported that cervical cancer affected 493,243 women worldwide in 2002 (1). It remains a health threat with estimated incidence and mortality rates of 12,710 and 4,290 in 2011, respectively, in the United States (2). Cervical cancer is now considered a preventable disease (3), but it is important to note that cervical cancer affects young women at a higher incidence. Treatment paradigms in the primary management of cervical cancer are well established. Early stage patients are treated surgically and women with locally advanced disease are managed with concomitant cisplatin chemoradiotherapy. However, the prognosis of patients with metastatic, recurrent, or persistent cervical cancer remains poor with a 1-year survival rate between $15-20 \%$ (4). In addition, chemotherapy has not led to major improvements in clinical outcome and is associated with high rates of severe toxicities. Advanced cervical cancer is associated with significant morbidities such as renal failure, complex fistulas and painful bone metastases. Therefore, improvement of systemic chemotherapy is crucial and new regimens should be further developed.

Persistent infection with an oncogenic-type human papillomavirus (HPV) is thought to be a prerequisite for the development of cervical cancer. The two HPV oncogenes, E6 and E7, are required for efficient immortalization of primary epithelial keratinocytes. The E6 proteins form a complex with p53 $(5,6)$, and subsequent disruption of multiple functions of p53 is an important step in cervical carcinogenesis (7). E6 also affects the function of tumor suppressors involved in apoptosis, cell cycle regulation, and tissue polarity, including two human homologues of Drosophila neoplastic tumor suppressors, hDlg and hScrib $(8,9)$.

Recent evidence indicates that rapidly proliferating cells, particularly cancer cells, have a greater requirement for proteasomal activity and a greater sensitivity to the proteasome inhibitor compared to normal cells (10). Bortezomib, a proteasome inhibitor, has been approved by the United 
States Food and Drug Administration for the treatment of multiple myeloma, and bortezomib has shown in vitro and in vivo activity against solid tumors, including prostate, pancreatic and colon cancer (11). In gynecologic cancers, several investigators have reported implicative roles of bortezomib for the treatment of human cervical (12-16) and ovarian cancer (17).

In this study, we report that sequential or concomitant use of bortezomib with cisplatin markedly induces apoptosis and inhibition of growth in cultured cervical cancer cells and xenograft. Bortezomib may have pre-clinical activity in cisplatin-resistant tumors and may have synergic activity when combined with cisplatin in HeLa cells. These effects may have an important clinical implication to maximize the stabilization of tumor suppressors (18).

\section{Materials and methods}

Chemicals and antibodies. Bortezomib (VELCADE, formerly known as PS-341) was kindly provided by Millennium Pharmaceuticals (Cambridge, MA, USA). Cisplatin, carboplatin and paclitaxel were from Bristol-Myers Squibb (Princeton, NJ, USA). Bortezomib, cisplatin, carboplatin and paclitaxel were dissolved in dimethyl sulfoxide and the final concentration of dimethyl sulfoxide never exceeded $0.05 \%$.

Anti-hScrib, anti-pRb (Ser 795), anti-p53 and anti-p21 were purchased from Santa Cruz Biotechnology (Santa Cruz, CA, USA). Rabbit polyclonal antibody was anti-Noxa (AnaSpec, Inc., San Jose, CA, USA). Mouse monoclonal antibody was anti- $\alpha$-Tubulin (Calbiochem, EMD Biosciences, Inc., La Jolla, CA, USA). Alexa Fluor 488-conjugated donkey anti-mouse IgG (A-21202) and Alexa Fluor 555-conjugated goat anti-rabbit IgG (A-21428) were purchased from Invitrogen (Carlsbad, CA, USA).

Cell culture. HeLa (CCL-2) and CaSki (HB-8307) uterine cervical cancer cell lines were purchased from the American Type Culture Collection (Manassas, VA, USA) and grown in DMEM supplemented with $10 \%$ fetal bovine serum.

Sequential and simultaneous treatment regimens. To determine the effect of sequence difference on cellular response, cells were treated with bortezomib $(100 \mathrm{nM})$, carboplatin $(250 \mu \mathrm{M})$, paclitaxel $(10 \mu \mathrm{M})$ and cisplatin $(500 \mu \mathrm{M})$. HeLa and CaSki cells were seeded and allowed to adhere for $24 \mathrm{~h}$. For the sequential treatment, after $12 \mathrm{~h}$ of initial treatment, the medium was changed to fresh medium containing the other treatment. For the simultaneous treatment, after an initial $12 \mathrm{~h}$ in medium, cells were treated with fresh medium containing the same drugs. The control cell medium was changed at similar time points. After the second 12-h treatment, the cells were harvested for flow cytometric analysis or western blotting. Therefore, all groups received the same duration of exposure to each agent and the assays were performed at the same point following the final treatment.

Cell viability test. Viability of HeLa and CaSki cells was examined using the CellTiter 96 Aqueous One Solution Cell Proliferation Assay kit (Promega Corp., Madison, WI, USA), as previously described (19).
Pulse chase analysis of $p 53$ and hScrib. The culture medium of HeLa cells was replaced with Met/Cys-free DMEM for $2 \mathrm{~h}$ and pulsed with $20 \mu \mathrm{Ci} / \mathrm{ml}$ of EasyTag ${ }^{\mathrm{TM}}$ EXPRESS $^{35} \mathrm{~S}$ (Perkin-Elmer Life Sciences, Boston, MA, USA) for $1.5 \mathrm{~h}$. The ${ }^{35} \mathrm{~S}$-labeled protein was chased with or without bortezomib $(100 \mathrm{nM})$. Cells were harvested at the indicated time point, lysed and electrophoresed.

Western blotting. Cultured cells and mouse xenograft tissues were harvested and soluble protein was extracted. The procedure of western blotting and subsequent immunoblot was performed as previously described (20).

Flow cytometric analysis. To determine the apoptosis rate, cells were grown in DMEM and treated with chemotherapeutic drugs. Bortezomib, cisplatin, carboplatin and paclitaxel treatments in 12-h increments over a 24 -h period were as described. Following treatment, cells were harvested and stained with Annexin V-FITC and propidium iodide (PI) according to the manufacturer's protocol (BD Biosciences, Bedford, MA, USA). The percentage of specific apoptosis was analyzed on FACSCalibur and calculated by CELLQuest Pro software (BD Biosciences).

Quantification of the synergism of bortezomib with cisplatin in the manner of sequential or simultaneous administration (Chou-Talalay assay). For the Chou-Talalay assay, experiments were carried out as previously described (21). Dose-response curves and $50 \%$ effective dose values $\left(\mathrm{ED}_{50}\right)$ were obtained, and fixed ratios of drugs and mutually exclusive equations used to determine combination indices (CI) (22). The potency of the combination was calculated with the CalcuSyn software (Biosoft, Ferguson, MO, USA). $\mathrm{CI}<1, \mathrm{CI}=1$, and $\mathrm{CI}>1$ indicate synergistic, additive and antagonistic interactions, respectively.

Tumor growth suppression in vivo. Athymic C.B-17/Icr-scid Jcl mice 5-7 weeks of age (CLEA Japan, Inc., Tokyo, Japan) were maintained in an SPF facility according to the institutional guidelines, and experiments were conducted under an approved animal protocol of The University of Tokyo. Subcutaneous xenograft tumors were established by the injection of cell suspension of $1 \times 10^{7} \mathrm{HeLa}$ and CaSki cells. After the appropriate tumors were formed, the mice were sacrificed. The tumors were removed, cut into 3-mm sections and transplanted subcutaneously into other mice. Mice were randomly assigned to one of the four treatment regimens: saline (control), cisplatin [intraperitoneal (i.p.) injection of cisplatin at a dose of $6 \mathrm{mg} / \mathrm{kg}$ in a volume of $0.5 \mathrm{ml}$ (23)], bortezomib [i.p. injection of bortezomib at a dose of $1 \mathrm{mg} / \mathrm{kg}$ in a volume of $0.5 \mathrm{ml}(24)$, and bortezomib followed by cisplatin $8 \mathrm{~h}$ later in a combined volume of $0.5 \mathrm{ml}$. Each treatment group consisted of 6 mice. Tumors were measured and the volume of these tumors was calculated using the formula; Volume $\left(\mathrm{mm}^{3}\right)=[$ (major axis $)$ $\mathrm{x}$ (minor axis) $\left.{ }^{2}\right] / 2$. After 4 weeks of treatment, the mice were sacrificed and subjected to the analysis.

Immunofluorescence. The mouse xenograft tumors were frozen in OCT compounds (Sakura Finetek Japan Co., Ltd., Tokyo, Japan). The embedded tissues were cut $(6 \mu \mathrm{m})$ and cryostat sections were recovered and fixed with PBS containing 


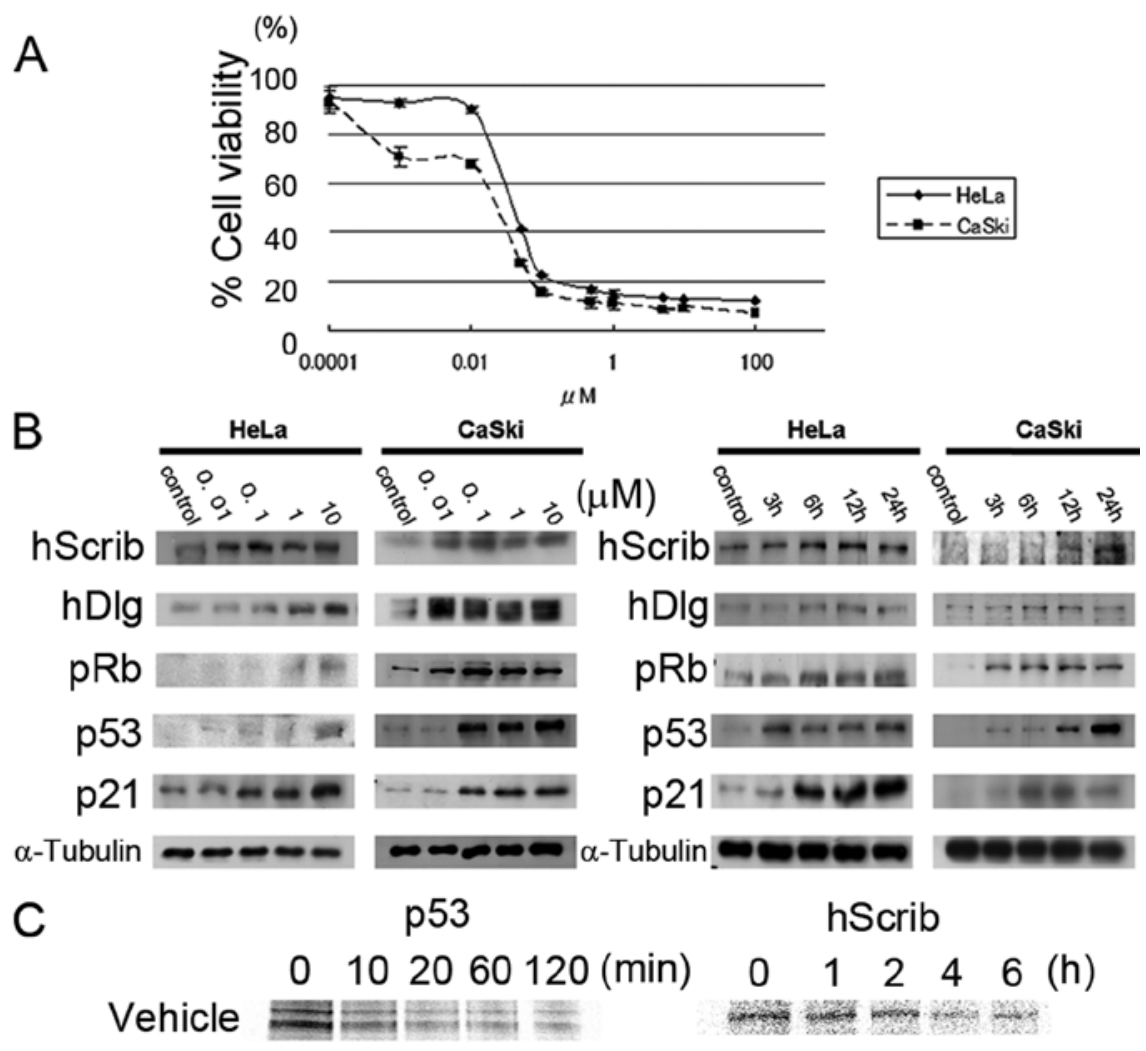

\section{Bortezomib $\equiv \equiv \equiv \equiv \equiv$}

Figure 1. Biological effects of bortezomib on human cervical cancer cell lines and stabilization of tumor suppressors. (A) HeLa and CaSki cells were exposed to bortezomib for $48 \mathrm{~h}$ and cell viability was determined by MTS assay. Each point represents the means \pm SD of 3 independent experiments. (B) The effect of bortezomib on the expression of tumor suppressor proteins. (1) HeLa and CaSki cells were exposed to various concentrations of bortezomib for $12 \mathrm{~h}$ (left panel). (2) Cells treated by $100 \mathrm{nM}$ of bortezomib were harvested at the indicated time points (right panel). The expression levels of hScrib, hDlg, pRb, p53 and p21 were analyzed by western blotting. (C) Pulse chase analysis of p53 and hScrib. p53 and hScrib proteins were ${ }^{35} \mathrm{~S}$-labeled in HeLa cells and the amount of labeled proteins were chased in the presence and absence of bortezomib. In the presence of bortezomib, the expression of p53 and hScrib remained unaffected during the observed time.

$4 \%$ paraformaldehyde. After blocking, the cells were sequentially incubated with anti-p53 or anti-hScrib antibodies and appropriate secondary antibodies. The slides were briefly counterstained and analyzed under the fluorescence microscope (Olympus BX50; Olympus, Tokyo, Japan). Apoptotic cells were detected by DeadEnd ${ }^{\mathrm{TM}}$ Fluorometric TUNEL System (Promega Corp.) in the mouse xenograft tumors.

Statistical analysis. Data represent the means \pm SD or SEM from at least 3 independent experiments. Statistical analyses were performed by one-way ANOVA with post-hoc test for multiple comparisons by using StatView software (SAS Institute Inc., Cary, NC, USA). A P-value $<0.05$ was considered to indicate statistically significant differences.

\section{Results}

Effect of bortezomib on cellular viability and stabilization of tumor suppressor proteins targeted for degradation by HPV E6 and E7 in human cervical cancer cell lines. We used MTS assay to determine the effect of bortezomib on cell viability in HeLa and CaSki cells (Fig. 1A). The approximately estimated $\mathrm{IC}_{50}$ of bortezomib in HeLa and CaSki cells was $100 \mathrm{nM}$. The expression of p53 increased by the exposure to bortezomib in a dose-dependent manner (Fig. 1B left panel) and in a time-dependent manner (Fig. 1B right panel). As a result, the expression of p21 also increased. Elevated expression of PDZ domain-containing scaffolding proteins (hScrib and $\mathrm{hDlg}$ ) was shown by the exposure to bortezomib (Fig. 1B). The expression of $\mathrm{pRb}$ decreases by the proteasome system during cervical carcinogenesis (25), and the expression of pRb was remarkably stimulated by the addition of bortezomib, particularly in CaSki cells (Fig. 1B). These data were translated as the antitumorigenic properties of bortezomib and we further confirmed whether p53 and hScrib are stabilized in the presence of bortemozib by the pulse-chase analysis. As expected, p53 and hScrib expression remained unaffected in the presence of bortezomib in HeLa cells (Fig. 1C).

Effect of bortezomib and chemotherapeutic agents on cervical cancer cells. The effect of bortezomib and chemotherapeutic drugs on the induction of apoptosis was analyzed by the flow cytometric analysis (Fig. 2A). As a single agent, bortezomib (lane 2) possessed a significant ability to induce apoptosis compared to cisplatin, carboplatin, and paclitaxel (lanes 3, 4 and 5, respectively), and the rate of apoptosis was superior in CaSki cells. The sequential and simultaneous combination of bortezomib with cisplatin was identified to be the most 

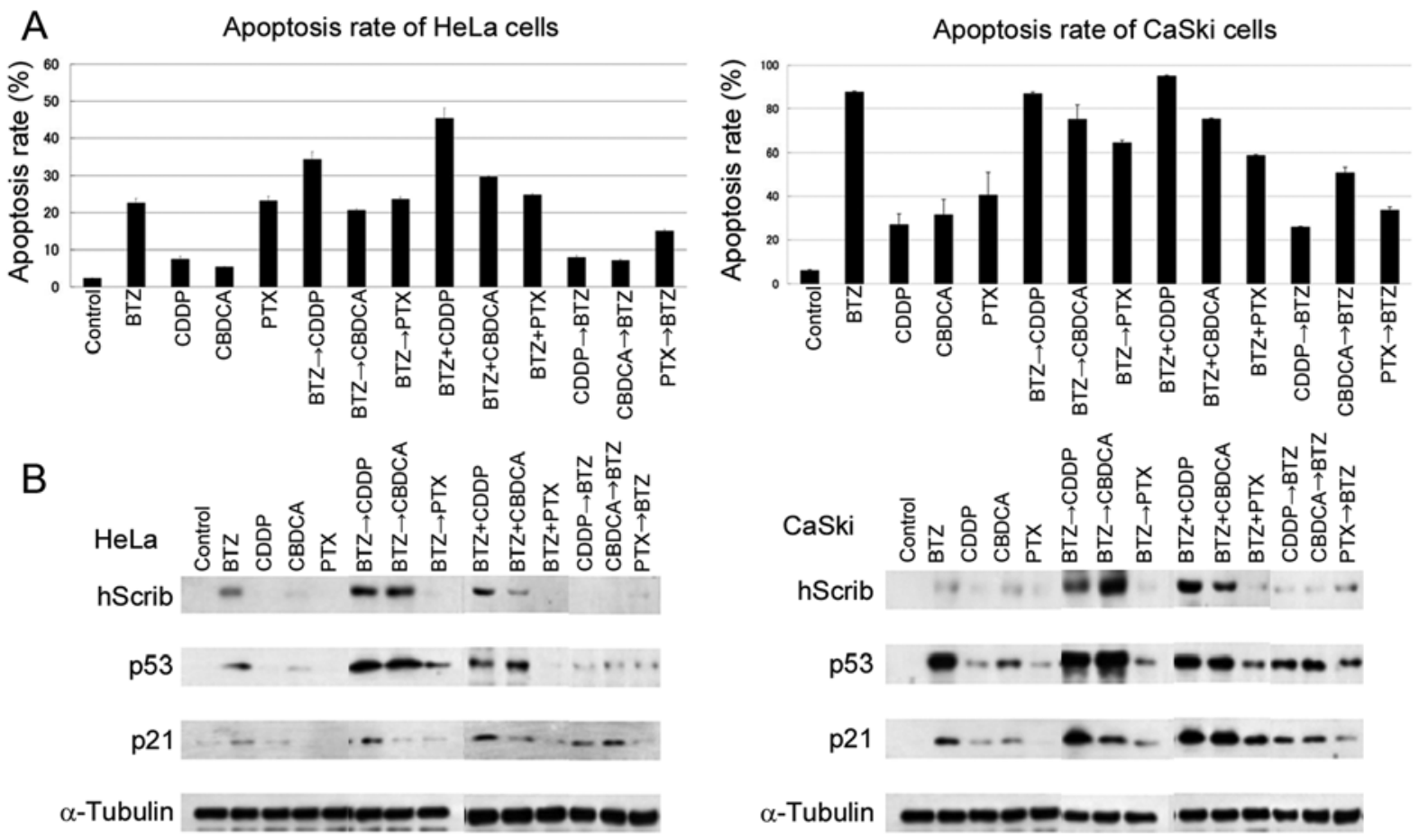

C

Synergy test (HeLa cell)

\begin{tabular}{|c|c|c|c|c|}
\hline $\begin{array}{l}\text { CDDP } \\
\text { (mM) }\end{array}$ & $\begin{array}{l}\text { BTZ } \\
\text { (uno }\end{array}$ & $\stackrel{\mathrm{BT}}{\mathrm{BTZ}}$ & $\begin{array}{l}\text { cI } \\
\text { BTZ } \\
+ \text { CDDP }\end{array}$ & $\begin{array}{l}\text { C1 } \\
\text { CDDP } \\
\rightarrow \text { BTZ }\end{array}$ \\
\hline 0.25 & 0.0001 & 0.142 & 0.108 & 1316 \\
\hline 25 & 0.000 & 0.117 & 0.013 & 1.197 \\
\hline 25 & $0.0 \mathrm{n}$ & 0.111 & 0.008 & 3515 \\
\hline 250 & 0.1 & 0.10 & 0.021 & 3.65 \\
\hline 2500 & 1 & 0.05 & 0.008 & 16065 \\
\hline
\end{tabular}

\begin{tabular}{|c|c|c|c|c|}
\hline $\begin{array}{l}\text { CDDP } \\
\text { (mM) }\end{array}$ & $\begin{array}{l}\text { BTZ } \\
\text { (uM) }\end{array}$ & $\begin{array}{l}\text { Cl } \\
\text { BTZ } \\
\rightarrow \text { CDDP }\end{array}$ & $\begin{array}{l}\text { Cl } \\
\text { BTZ } \\
+ \text { +CDDP }\end{array}$ & $\begin{array}{l}\text { CI } \\
\text { CDDP } \\
\rightarrow \text { BTZ }\end{array}$ \\
\hline 025 & 0.0001 & 0819 & 0.607 & 2.112 \\
\hline 25 & 0.001 & 0548 & 0204 & 7237 \\
\hline 25 & 0.01 & 0337 & 0336 & 23606 \\
\hline 250 & 0.1 & 0.572 & 0.191 & 11961 \\
\hline 2500 & 1 & 0.4 & 0235 & 1043 \\
\hline
\end{tabular}

Figure 2. An analysis of bortezomib and chemotherapeutic drugs in cervical cancer cells. (A) The rate of apoptosis using bortezomib and chemotherapeutic drugs was measured by flow cytometric analysis. Single, sequential, or simultaneous treatment regimens using bortezomib $(100 \mathrm{nM})$, cisplatin $(500 \mu \mathrm{M})$, carboplatin $(250 \mu \mathrm{M})$ or paclitaxel $(10 \mu \mathrm{M})$ were applied for HeLa and CaSki cells. Bortezomib followed by cisplatin and concomitant bortezomib with cisplatin regimens induced higher apoptosis rates compared with other regimens. Bars represent the means \pm SD of 3 independent experiments. (B) Bortezomib stabilized the expression of hScrib and p53 in cervical cancer cell lines in combination with platinum agents. By contrast, cisplatin followed by bortezomib failed to stimulate the expression of hScrib, p53 and p21. (C) Quantification of the potency of the combination treatment. The Chou-Talalay method was utilized. Bortezomib followed by cisplatin and concomitant bortezomib with cisplatin regimens exhibited synergistic effects in all the doses tested. A potent synergistic effect was displayed with $\mathrm{CI}<0.1$. By contrast, a low synergistic or antagonistic effect was shown in the treatment using cisplatin followed by bortezomib with CI>1. BTZ, bortezomib; CDDP, cisplatin; CBDCA, carboplatin; PTX, paclitaxel.

significant treatment regimens in inducing cellular apoptosis (Fig. 2A, lanes 6 and 9) in HeLa cells.

We further investigated the expression of hScrib, p53 and p21, whether the apoptotic effects are indeed associated with protein expressions. Cells were exposed to various concentrations of bortezomib and/or chemotherapeutic drugs (Fig. 2B). The elevated expression of p53 was observed in HeLa cells treated with bortezomib followed by cisplatin or carboplatin (lanes 6 and 7), and in CaSki cells treated with bortezomib alone (lane 2), bortezomib followed by cisplatin or carboplatin (lanes 6 and 7). The expression of hScrib exhibited a similar tendency to that of p53. In terms of the stabilization of p53 and hScrib, paclitaxel turned out to be an unsatisfactory agent (lanes 5, 8, 11 and 14). Markedly, bortezomib and subsequent cisplatin treatment was the most efficient regimen to induce the expression of p21, particularly in CaSki cells (lane 6). Cisplatin followed by bortezomib treatment had an insignificant effect on the expression of p53 and hScrib (lane 12). The apparent recovery of $\mathrm{pRb}$ was also observed in HeLa cells treated by the exposure to bortezomib followed by cisplatin (data not shown).

The synergistic effects of bortezomib and cisplatin were analyzed using the Chou-Talalay assay. Bortezomib followed by cisplatin and simultaneous bortezomib with cisplatin regimens were synergistic, with $\mathrm{CI}<1$. This synergy was evidently affected by the order of addition as cisplatin followed by bortezomib showed antagonistic interactions, with $\mathrm{CI}>1$. Therefore, the synergistic effects in HeLa cells was more pronounced compared to that in CaSki cells and we determined to pursue the sequential effect of bortezomib and cisplatin.

In vivo suppression of cervical cancer growth by bortezomib in a mouse xenograft. We analyzed whether bortezomib is able to inhibit tumor growth using a xenograft mouse model of cervical cancer cells. Bortezomib was administered twice a 


\section{A HeLa xenograft}
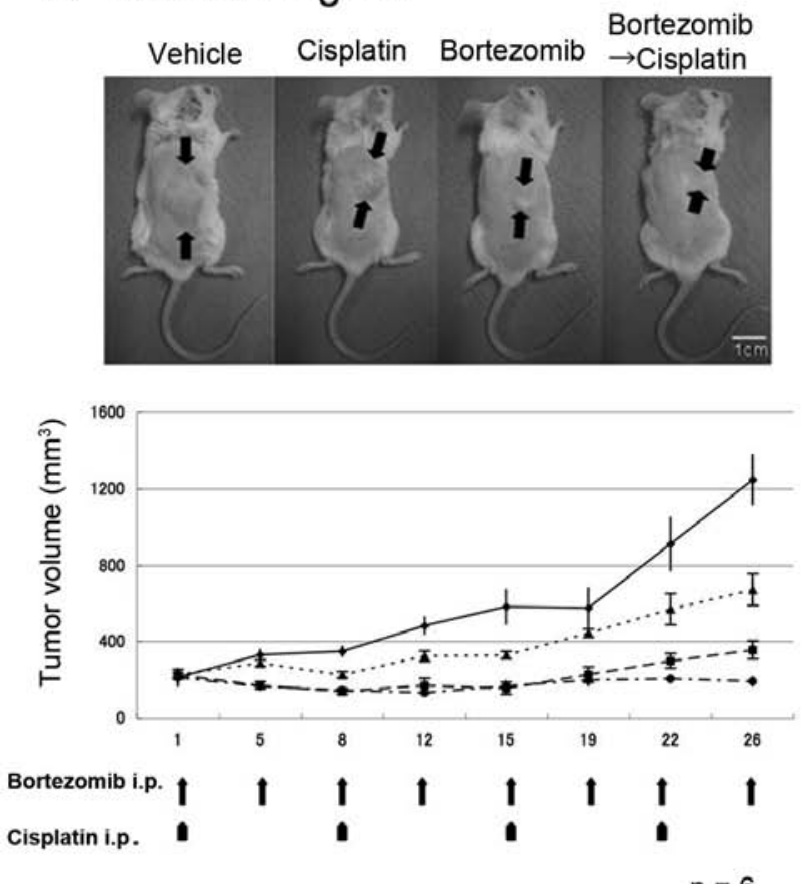

\section{B CaSki xenograft}
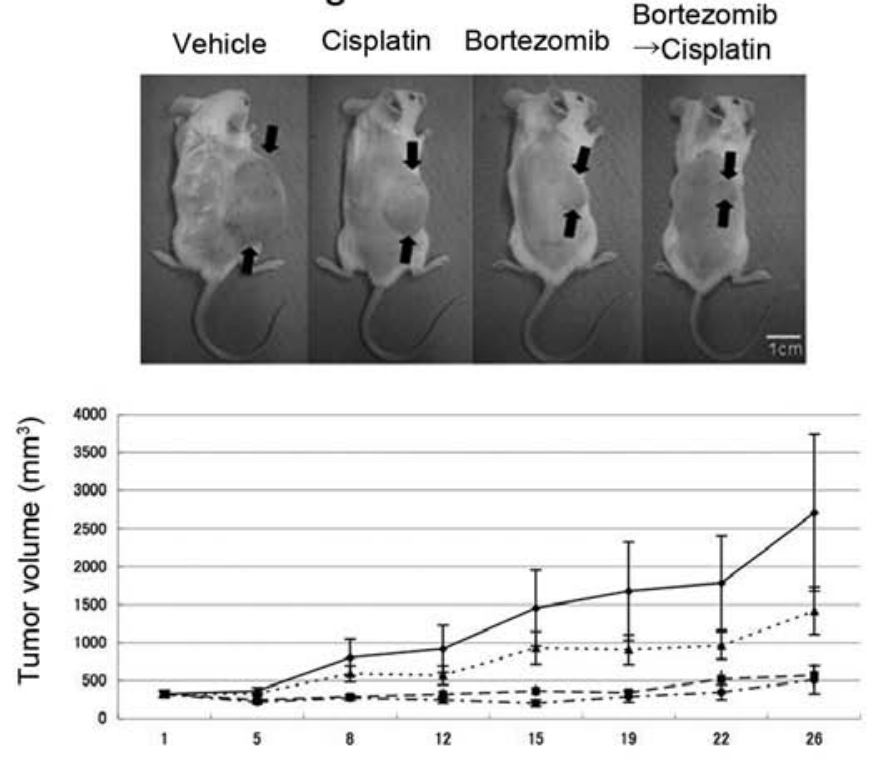

Bortezomibi.p. 1 I 1 1 1 1 1 1

Cisplatin i.p.

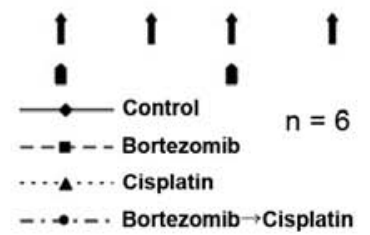

Figure 3. Bortezomib and bortezomib with cisplatin efficiently inhibited the growth of cervical cancer xenografts in athymic mice. (A) Representative images of xenograft mice are shown. Bars indicate $1 \mathrm{~cm}$. (B) HeLa and CaSki cells were transplanted into 6 to 7-week-old athymic mice. When xenograft tumors were visualized, 4 treatment regimens were commenced. Treatment schedules are indicated by thin arrows (bortezomib) and thick arrows (cisplatin). The dose of bortezomib was $1 \mathrm{mg} / \mathrm{kg}$, and the dose of cisplatin was $6 \mathrm{mg} / \mathrm{kg}$, according to the recommendation of the suppliers. Each treatment group consisted of 6 mice. Tumor volumes were calculated as described in Materials and methods. Bars indicate the means \pm SEM.

week, and cisplatin once a week according to the instructions of the suppliers. As shown in Fig. 3, the tumor volume of the HeLa and CaSki xenograft was significantly reduced by the injection of bortezomib when compared with the control. The reduction rate of the tumor growth in the bortezomib group was greater than that of cisplatin alone and we revealed that bortezomib with cisplatin was the most efficient treatment regimen in the HeLa xenograft. The CaSki xenograft exhibited a pronounced sensitivity to bortezomib alone and the effect of concomitant use was not observed as expected from the in vitro study (Fig. 2, right panel).

Based on the data in Fig. 2, the expression levels of p53, Noxa, and p21 in cervical cancer xenografts were analyzed by western blot analysis. It became evident that p53 and its downstream genes are upregulated in xenografts treated by bortezomib (Fig. 4A and B). Our immunofluorescence study also revealed that the expression of $\mathrm{hScrib}$ was recovered at the cellular membrane in mice treated with bortezomib alone or in combination with cisplatin (Fig. 4C and D). The expression of p53 in the nuclei of xenograft cells also increased as a result of treatment of bortezomib (Fig. 4C and D). HeLa and CaSki xenografts were subjected to the detection of apoptosis using TUNEL assay and TUNEL positive cells were observed predominantly in xenograft tumors treated by bortezomib (Fig. 4C and D). These data suggest the possibility that bortezomib inhibits tumor growth in vivo through the induction of apoptosis driven by p53 and downstream genes of p53, particularly in HeLa cells.

\section{Discussion}

Bortezomib has been shown to be an extremely potent, reversible and selective proteasome inhibitor (26). Several investigators have demonstrated the ability of bortezomib to sensitize a variety of cancer cells to the apoptotic effects of diverse chemotherapeutic agents (12-15,17,27-29). We confirmed that bortezomib was able to induce apoptosis in HPV-positive cervical cancer cell lines. Cisplatin, a critical component of therapeutic regimens in a broad range of malignancies, has been shown to induce apoptosis in various types of cancer cells. For the treatment of advanced or recurrent cervical cancer, cisplatin administered every 3 weeks seems to be a reasonable option, inducing response rates ranging from 20 to $30 \%$ and an overall survival of 7 months (30), but prolonged cisplatin treatment appears to have considerable side-effects. We aimed to explore the possibility that concomitant use of bortezomib and chemotherapeutic drugs has additive effects to suppress ubiquitin-mediated degradation of tumor suppressors targeted by E6 in HPV-positive cervical cancer cells both in vitro and in vivo. As expected, bortezomib alone increased the expression of tumor suppressors such as p53 and hScrib in a dose- and time-dependent manner. We also observed the elevated expression of $\mathrm{p} 21$ by the treatment of bortezomib since p21 is a representative downstream gene of p53. We noted that the rate of apoptosis in cells treated with bortezomib preceded by cisplatin was extremely lower than that in cells treated with cisplatin preceded by bortezomib in HeLa cells. The sequential 

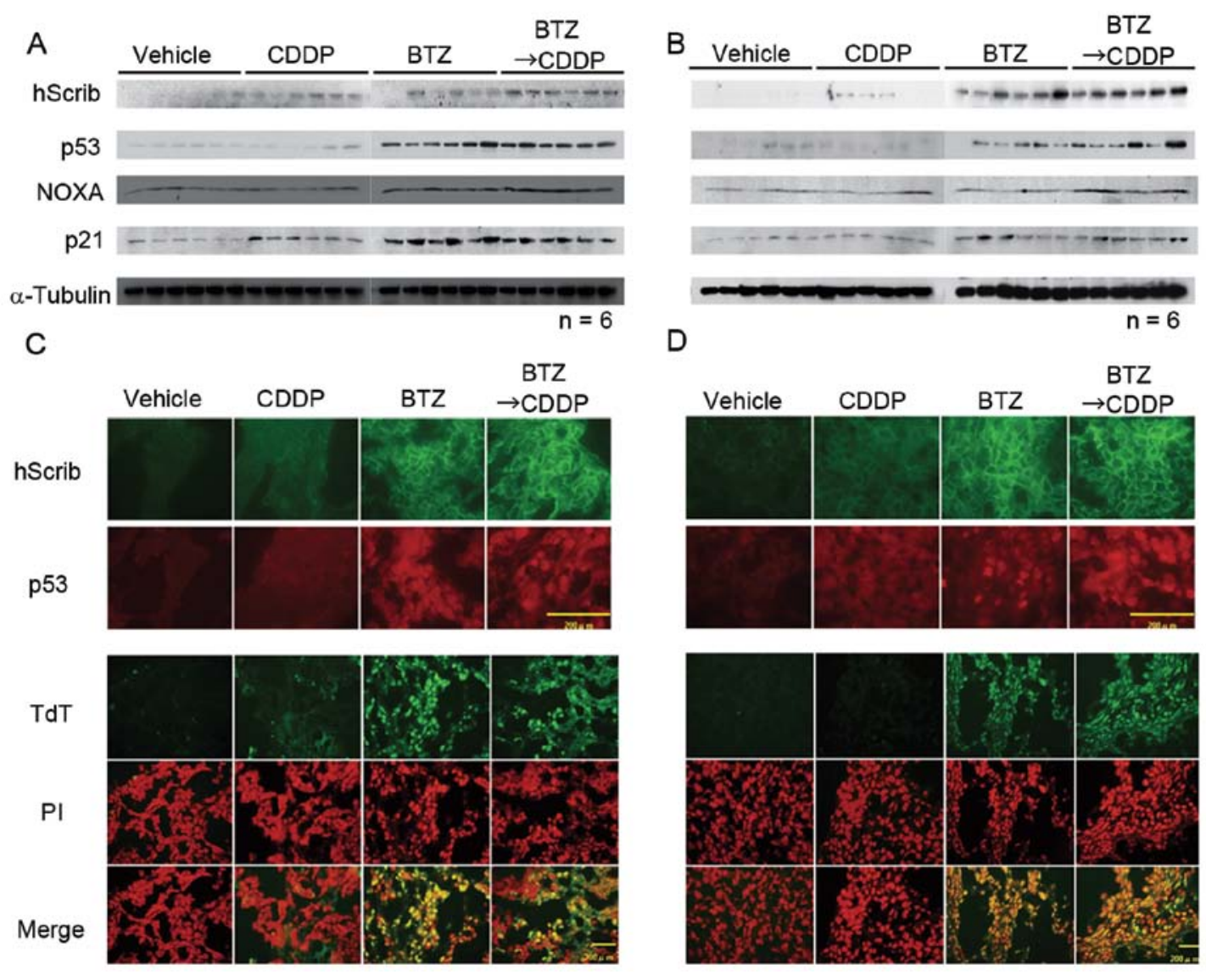

Figure 4. The growth inhibitory effect of bortezomib and cisplatin is evidenced by elevated expression of p53 and hScrib in xenografts of athymic mice. (A and B) Whole cell extracts were obtained from xenograft tumors and the expression levels of hScrib, p53, Noxa and p21 were analyzed by western blotting. (A) HeLa cell xenograft; (B) CaSki cell xenograft. (C and D) The expression levels of hScrib and p53 were visualized by immunofluorescence (upper panel). TUNEL assay revealed that bortezomib and concomitant use of bortezomib and cisplatin induced significant apoptosis in xenograft tumors (lower panel). (C) HeLa cell xenograft; (D) CaSki cell xenograft. Bars indicate $200 \mu \mathrm{m}$.

effect (bortezomib followed by cisplatin) on the induction of apoptosis was prominent compared with other combinations. However, this sequential effect was less pronounced in CaSki cells both in vitro and in vivo, and was similar to a previous report that bortezomib did not induce a sensitization to cisplatin treatment in SiHa cells (16). We must take into account the fact that bortezomib alone was able to induce significant apoptosis in CaSki cells (Fig. 2A, right panel), and this result is in concordance with the result of the Chou-Talalay assay that synergistic effects in HeLa cells were more pronounced compared to those in CaSki cells (Fig. 2C). The optimal sequence of chemotherapies with disparate mechanisms of action has been investigated intensively. Although the mechanisms of the sequence-specific interactions with chemotherapy remain unclear, one possible explanation could be the effect on the apoptotic mechanism by bortezomib. Cisplatin was shown to have a defect in mitochondria-dependent caspase- 9 activation in non-small cell lung cancer H460 cells (29). Contrary to this, bortezomib efficiently induced caspase- 9 activation and apoptosis by promoting a pro-apoptotic shift in the levels of proteins involved in mitochondrial outer-membrane permeabilization (29). Another mechanism might be its distribution to cell cycle arrest. Bortezomib causes G2/M arrest (31) and cisplatin causes long-lasting blocks at the G1/S boundary with increasing cytotoxicity (32). Theoretically, when bortezomib is administered first, the increased p53 may serve to enhance G1/S checkpoint function. Then G1/S arrest by cisplatin may efficiently enhance apoptotic cell death.

We further examined whether concomitant use of bortezomib and cisplatin can induce apoptosis in vivo. Although the regimen was not completely identical to that of the in vitro experiment, the concomitant use of bortezomib and cisplatin abrogated the tumor growth of xenografts. In bortezomibtreated tumors, p53 is apparently stabilized in the nuclei of tumor cells, and, subsequently, p21 and Noxa are elevated due to the increased expression of p53. TUNEL assay showed enhanced apoptosis in xenografts treated by bortezomib alone or bortezomib with cisplatin, but concomitant treatment showed significant enhancement of apoptosis. Several investigators have reported the sequence-dependent effects of bortezomib are limited $(28,33)$. The optimal apoptotic effect occurs with the sequence gemcitabine followed by bortezomib in pancreatic cancer cells (33) and in lung cancer cells (28). Therefore, the effect of bortezomib may be sensitization of cancer cells to the apoptotic effect and may be modulating the cellular response to the chemotherapeutics. As a result, bortezomib may enhance cell death in combination with cisplatin. Our data provide new evidence that the schedule of combination treatment must be considered for the treatment of cervical cancer, and gynecologists should consider pre-clinical data in 
the design of clinical trials. While it is important to confirm sequential effects in each cancer type studied, it appears that chemotherapy given prior to bortezomib may yield inferior results.

In conclusion, a proteasome inhibitor, bortezomib, induces apoptosis in HPV-positive cervical cancer cells depending on the stabilization of tumor suppressors, particularly p53. When bortezomib is combined with cisplatin, a higher effect of apoptosis induction might be expected since bortezomib plus cisplatin almost completely abolished growth of cervical cancer xenografts with the recovery of p53 and hScrib expression. Our data suggest the possibility that bortezomib plus cisplatin is a promising regimen for the treatment of advanced and/or chemotherapy or radiation therapy-resistant cervical cancer cases.

\section{Acknowledgements}

This study was supported by the Mitsui Life Social Welfare Foundation and the grant-in-aid for Scientific Research from the Ministry of Education, Science and Culture, Japan.

\section{References}

1. Parkin DM, Bray F, Ferlay J and Pisani P: Global cancer statistics, 2002. CA Cancer J Clin 55: 74-108, 2005.

2. American Cancer Society: Cancer Facts and Figures 2011. http://www.cancer.org/Cancer/CervicalCancer. 2011.

3. Carter JR, Ding Z and Rose BR: HPV infection and cervical disease: a review. Aust N Z J Obstet Gynaecol 51: 103-108, 2011.

4. Berek JS and Hacker NF (eds): Practical Gynaecologic Oncology. 4th edition. Lippincott Williams \& Wilkins, Philadelphia, PA, 2005.

5. Nakagawa S, Watanabe S, Yoshikawa H, Taketani Y, Yoshiike K and Kanda T: Mutational analysis of human papillomavirus type 16 E6 protein: transforming function for human cells and degradation of p53 in vitro. Virology 212: 535-542, 1995.

6. Crook T, Tidy JA and Vousden KH: Degradation of p53 can be targeted by HPV E6 sequences distinct from those required for p53 binding and trans-activation. Cell 67: 547-556, 1991.

7. Huibregtse JM and Beaudenon SL: Mechanism of HPV E6 proteins in cellular transformation. Semin Cancer Biol 7: 317-326, 1996.

8. Massimi P, Gammoh N, Thomas M and Banks L: HPV E6 specifically targets different cellular pools of its PDZ domain-containing tumour suppressor substrates for proteasome-mediated degradation. Oncogene 23: 8033-8039, 2004.

9. Nakagawa S and Huibregtse JM: Human scribble (Vartul) is targeted for ubiquitin-mediated degradation by the high-risk papillomavirus E6 proteins and the E6AP ubiquitin-protein ligase. Mol Cell Biol 20: 8244-8253, 2000.

10. Mani A and Gelmann EP: The ubiquitin-proteasome pathway and its role in cancer. J Clin Oncol 23: 4776-4789, 2005.

11. Adams J and Kauffman M: Development of the proteasome inhibitor Velcade (Bortezomib). Cancer Invest 22: 304-311, 2004

12. Birle DC and Hedley DW: Suppression of the hypoxia-inducible factor-1 response in cervical carcinoma xenografts by proteasome inhibitors. Cancer Res 67: 1735-1743, 2007.

13. Kamer S, Ren Q and Dicker AP: Differential radiation sensitization of human cervical cancer cell lines by the proteasome inhibitor velcade (bortezomib, PS-341). Arch Gynecol Obstet 279: 41-46, 2009.

14. Lin Z, Bazzaro M, Wang MC, Chan KC, Peng S and Roden RB Combination of proteasome and HDAC inhibitors for uterine cervical cancer treatment. Clin Cancer Res 15: 570-577, 2009.
15. Jiang Y, Wang Y, Su Z, et al: Synergistic induction of apoptosis in HeLa cells by the proteasome inhibitor bortezomib and histone deacetylase inhibitor SAHA. Mol Med Rep 3: 613-619, 2010.

16. Bruning A, Vogel M, Mylonas I, Friese K and Burges A: Bortezomib targets the caspase-like proteasome activity in cervical cancer cells, triggering apoptosis that can be enhanced by nelfinavir. Curr Cancer Drug Targets 11: 799-809, 2011.

17. Frankel A, Man S, Elliott P, Adams J and Kerbel RS: Lack of multicellular drug resistance observed in human ovarian and prostate carcinoma treated with the proteasome inhibitor PS-341. Clin Cancer Res 6: 3719-3728, 2000.

18. Nagasaka K, Nakagawa S, Yano T, et al: Human homolog of Drosophila tumor suppressor Scribble negatively regulates cell-cycle progression from G1 to $\mathrm{S}$ phase by localizing at the basolateral membrane in epithelial cells. Cancer Sci 97: 1217-1225, 2006.

19. Morita Y, Wada-Hiraike O, Yano T, et al: Resveratrol promotes expression of SIRT1 and StAR in rat ovarian granulosa cells: an implicative role of SIRT1 in the ovary. Reprod Biol Endocrinol 10: 14, 2012.

20. Wada-Hiraike O, Yano T, Nei T, et al: The DNA mismatch repair gene hMSH2 is a potent coactivator of oestrogen receptor alpha. Br J Cancer 92: 2286-2291, 2005.

21. Chou TC: Drug combination studies and their synergy quantification using the Chou-Talalay method. Cancer Res 70: 440-446, 2010.

22. Kanai R, Wakimoto H, Martuza RL and Rabkin SD: A novel oncolytic herpes simplex virus that synergizes with phosphoinositide 3-kinase/Akt pathway inhibitors to target glioblastoma stem cells. Clin Cancer Res 17: 3686-3696, 2011.

23. Fraval HN and Roberts JJ: G1 phase Chinese hamster V79-379A cells are inherently more sensitive to platinum bound to their DNA than mid S phase or asynchronously treated cells. Biochem Pharmacol 28: 1575-1580, 1979.

24. Nawrocki ST, Bruns CJ, Harbison MT, et al: Effects of the proteasome inhibitor PS-341 on apoptosis and angiogenesis in orthotopic human pancreatic tumor xenografts. Mol Cancer Ther 1: 1243-1253, 2002.

25. Munger K and Howley PM: Human papillomavirus immortalization and transformation functions. Virus Res 89: 213-228, 2002.

26. Adams J: The proteasome: structure, function, and role in the cell. Cancer Treat Rev 29 (Suppl 1): 3-9, 2003.

27. Ling YH, Liebes L, Jiang JD, et al: Mechanisms of proteasome inhibitor PS-341-induced G(2)-M-phase arrest and apoptosis in human non-small cell lung cancer cell lines. Clin Cancer Res 9: 1145-1154, 2003.

28. Mortenson MM, Schlieman MG, Virudachalam S and Bold RJ: Effects of the proteasome inhibitor bortezomib alone and in combination with chemotherapy in the A549 non-small-cell lung cancer cell line. Cancer Chemother Pharmacol 54: 343-353, 2004.

29. Voortman J, Checinska A, Giaccone G, Rodriguez JA and Kruyt FA: Bortezomib, but not cisplatin, induces mitochondriadependent apoptosis accompanied by up-regulation of noxa in the non-small cell lung cancer cell line NCI-H460. Mol Cancer Ther 6: 1046-1053, 2007.

30. Tewari KS and Monk BJ: Gynecologic oncology group trials of chemotherapy for metastatic and recurrent cervical cancer. Curr Oncol Rep 7: 419-434, 2005.

31. Gregory MA and Hann SR: c-Myc proteolysis by the ubiquitinproteasome pathway: stabilization of c-Myc in Burkitt's lymphoma cells. Mol Cell Biol 20: 2423-2435, 2000.

32. Jackel M and Kopf-Maier P: Influence of cisplatin on cell-cycle progression in xenografted human head and neck carcinomas. Cancer Chemother Pharmacol 27: 464-471, 1991.

33. Fahy BN, Schlieman MG, Virudachalam S and Bold RJ: Schedule-dependent molecular effects of the proteasome inhibitor bortezomib and gemcitabine in pancreatic cancer. J Surg Res 113: 88-95, 2003. 